\title{
New Observations on Light Hadron Spectroscopy at BESIII
}

\section{Huang Yanping (for BESIII collaboration)*}

Institute of High Energy Physics, Beijing 100049, People's Republic of China

E-mail: huangypdihep.ac.cn

\begin{abstract}
With samples of 220 million $J / \psi$ events and 110 million $\psi^{\prime}$ events collected in the BESIII detector, $p \bar{p}$ mass threshold enhancement is studied. The enhancement is evident in $J / \psi$ radiative decay, which is consistent with BESII result. No significant narrow enhancement is observed in $\psi^{\prime}$ radiative decay. For $J / \psi \rightarrow \gamma \pi^{+} \pi^{-} \eta^{\prime}$ decay, the $X(1835)$, which was previously observed by BESII, is confirmed with a statistical significance that is larger than $20 \sigma$. In addition, in the $\pi^{+} \pi^{-} \eta^{\prime}$ invariant mass spectrum, the $X(2120)$ and the $X(2370)$, are observed with statistical significances larger than $7.2 \sigma$ and $6.7 \sigma$, respectively. A new process $J / \psi \rightarrow \omega X(1870) \rightarrow \omega a_{0} \pi$ is also observed in $J / \psi \rightarrow \omega \pi^{+} \pi^{-} \eta$ decay.
\end{abstract}

35th International Conference of High Energy Physics - ICHEP2010,

July 22-28, 2010

Paris France

${ }^{*}$ Speaker. 


\section{Introduction}

An anomalously strong $p \bar{p}$ mass threshold enhancement, the $X(1860)$, was observed by the BESII experiment in the radiative decay process $J / \psi \rightarrow \gamma p \bar{p}[1]$. An interesting feature of this enhancement is that corresponding structures are not observed in near-threshold $p \bar{p}$ cross section measurements, or in $B$-meson decays [2], or in radiative $\psi^{\prime}$ [3] or $\Upsilon \rightarrow \gamma p \bar{p}$ decays [4], or in $J / \psi \rightarrow \omega p \bar{p}$ decays [5]. One of theoretical speculations [6] is the intriguing suggestion that it is a $p \bar{p}$ bound state, sometimes called baryonium [6]. It also stimulated a study of $J / \psi \rightarrow \gamma \pi^{+} \pi^{-} \eta^{\prime}$, in which a resonance, the $\mathrm{X}(1835)$, was observed in the $\pi^{+} \pi^{-} \eta^{\prime}$ invariant-mass spectrum with a statistical significance of $7.7 \sigma$ at BESII [7]. The possible interpretations include a $p \bar{p}$ bound state [6], a glueball [8], a radial excitation of the $\eta^{\prime}$ meson [9], etc.

The high statistics data samples of $\sim 220 \times 10^{6} \mathrm{~J} / \psi$ and $\sim 110 \times 10^{6} \psi^{\prime}$ events accumulated by the upgraded Beijing Spectrometer (BESIII) in 2009, located at the Beijing Electron-Positron Collider (BEPCII) at the Beijing Institute of High Energy Physics, provide an opportunity to confirm the existence of above resonances, look for $0^{-+}$glueballs in $J / \psi \rightarrow \gamma \pi^{+} \pi^{-} \eta^{\prime}$ decays, and search for possible related states in other decay modes, such as $J / \psi \rightarrow \omega \pi^{+} \pi^{-} \eta$ decays.

\section{2. $p \bar{p}$ Mass Threshold Study in $J / \psi$ and $\psi^{\prime}$ radiative decays}
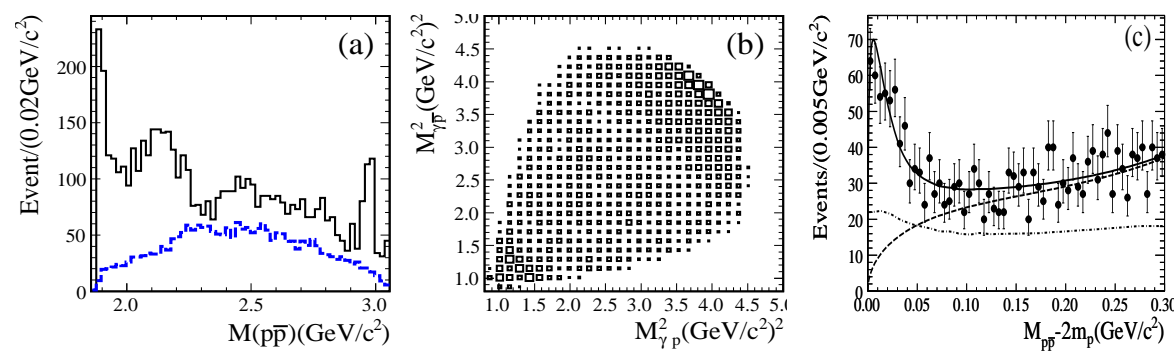

Figure 1: The selected $\psi^{\prime} \rightarrow \pi^{+} \pi^{-} J / \psi(J / \psi \rightarrow \gamma p \bar{p})$ events: (a) The $p \bar{p}$ invariant mass spectrum. (b) An $M^{2}(\gamma p)$ (horizontal) versus $M^{2}(\gamma \bar{p})$ (vertical) Dalitz plot for the selected events. (c) $p \bar{p}$ mass spectrum fitting in the threshold region, the solid curve is the fit result, the dashed curve shows the fitted background function, and the dash-dotted curve indicates how the acceptance varies with $M_{p \bar{p}}$.

Fig. 1(a) shows the $p \bar{p}$ invariant mass distribution for surviving events of $\psi^{\prime} \rightarrow \pi^{+} \pi^{-} J / \psi(J / \psi \rightarrow$ $\gamma p \bar{p})$. The distribution's features include the $\eta_{c}$ peak, a broad enhancement around $M_{p \bar{p}} \sim 2.2 \mathrm{GeV} / c^{2}$, and a prominent low-mass peak at the $p \bar{p}$ mass threshold, similar to that reported by BESII [1]. The Dalitz plot in Fig. 1(b) shows that a band corresponding to the threshold enhancement is evident in the upper right corner. Fitting with an acceptance weighted $S$-wave Breit-Wigner function plus the background shape shown in Fig. 1(c), yields a peak mass of $M=1861_{-13}^{+6}$ (stat $)_{-26}^{+7}$ (syst) $\mathrm{MeV} / c^{2}$ and a width of $\Gamma<38 \mathrm{MeV} / c^{2}$ at the $90 \%$ C.L.

The decay channel of $J / \psi \rightarrow \gamma p \bar{p}$ is also studied, the $p \bar{p}$ mass spectrum, as shown in Fig. 2(a)) has similar structure as Fig.1 (a)). In Fig. 2(b), the fitting in the $p \bar{p}$ mass spectrum with $S$-wave BW function can yield a peak mass of $M=1861.6 \pm 0.8$ (stat) $\mathrm{MeV} / c^{2}$ and a width of $\Gamma<8 \mathrm{MeV} / c^{2}$ at the $90 \%$ C.L. In the study of $\psi^{\prime} \rightarrow \gamma p \bar{p}$, there is no significant narrow threshold enhancement as 

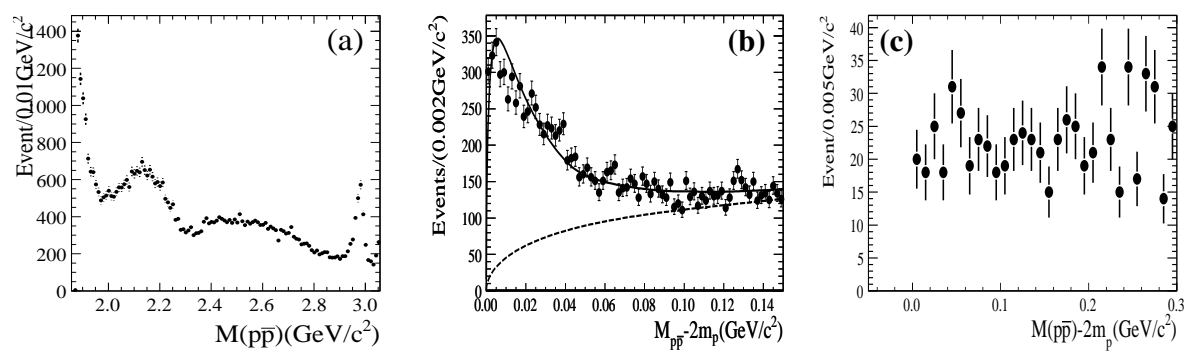

Figure 2: The $p \bar{p}$ invariant mass spectrum (a) and fitting in the threshold region (b) for the selected $J / \psi \rightarrow \gamma p \bar{p}$ events, where the solid curve is the fit result; the dashed curve shows the fitted background function. (c) is the $p \bar{p}$ invariant mass spectrum in the threshold region for the selected $\psi^{\prime} \rightarrow \gamma p \bar{p}$ events .

shown in Fig. 2(c). It indicates that the strong $p \bar{p}$ threshold enhancement observed in $J / \psi$ radiative decay disfavors the interpretation of pure final state interactions (FSI).

\section{Confirmation of $X(1835)$ and two new structures in $J / \psi \rightarrow \gamma \pi^{+} \pi^{-} \eta^{\prime}$ decays}
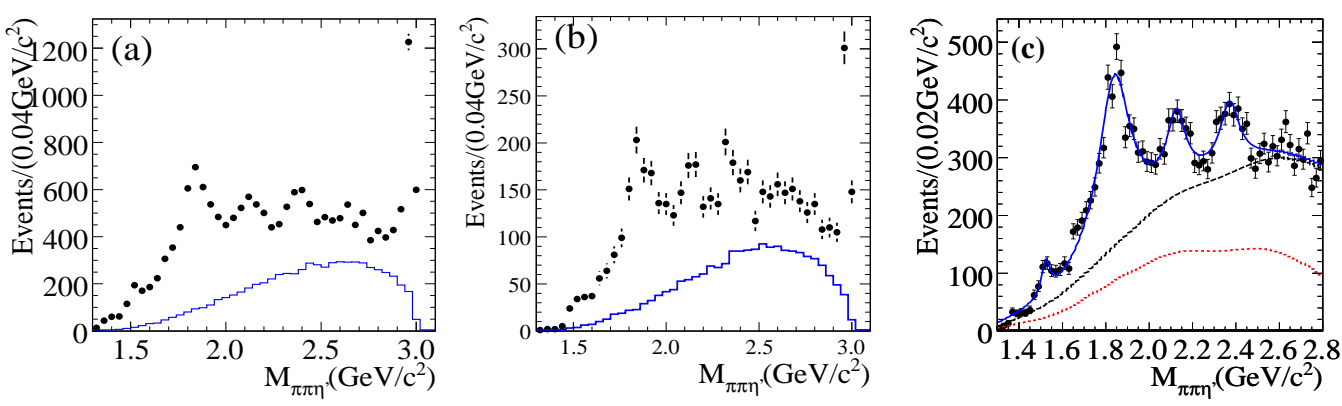

Figure 3: Invariant mass spectrum of $\pi^{+} \pi^{-} \eta^{\prime}$ after final selection for $J / \psi \rightarrow \gamma \pi^{+} \pi^{-} \eta^{\prime}\left(\eta^{\prime} \rightarrow \gamma \rho^{0}\right)$ (a) and $J / \psi \rightarrow \gamma \pi^{+} \pi^{-} \eta^{\prime}\left(\eta^{\prime} \rightarrow \pi^{+} \pi^{-} \eta, \eta \rightarrow \gamma \gamma\right)(\mathrm{b})$, where the solid circles are data and the histogram are from $J / \psi \rightarrow \gamma \pi^{+} \pi^{-} \eta^{\prime}$ phase space MC events(with arbitrary normalization). (c) is mass spectrum fitting with four resonances, the dash-dot line is contributions of non- $\eta^{\prime}$ events and the $\pi^{0} \pi^{+} \pi^{-} \eta^{\prime}$ background for two $\eta^{\prime}$ decay modes and the dash line is contributions of background and non-resonant $\pi^{+} \pi^{-} \eta^{\prime}$ process.

For $J / \psi \rightarrow \gamma \pi^{+} \pi^{-} \eta^{\prime}$ with the decay modes of $\eta^{\prime} \rightarrow \gamma \rho$ and $\eta^{\prime} \rightarrow \pi^{+} \pi^{-} \eta$, the $X(1835)$ resonance is clearly seen in the $\pi^{+} \pi^{-} \eta^{\prime}$ invariant mass spectrum of Fig. 3 (a) and (b). Additional peaks are evident around 2.1 and $2.4 \mathrm{GeV} / c^{2}$, the $X(2120)$ and $X(2370)$, as well as $f_{1}(1510)$ and a distinct signal for the $\eta_{c}$. The spectrum fits for the combined two $\eta^{\prime}$ decay modes have been made using four efficiency-corrected Breit-Wigner functions convolved with a Gaussian mass resolution plus non-resonant $\pi^{+} \pi^{-} \eta^{\prime}$ contribution and background representations shown in Fig.3(c). The statistical significance of the $X(1835)$ is larger than $20 \sigma$, while the $X(2120)$ and $X(2370)$, are larger than $7.2 \sigma$ and $6.7 \sigma$, respectively. The mass and width are $1838.1 \pm 2.8$ and $179.5 \pm 9.1$ $\mathrm{MeV} / c^{2}$ for the $X(1835), 2124.8 \pm 5.6$ and $101 \pm 14 \mathrm{MeV} / c^{2}$ for the $X(2120), 2371.0 \pm 6.4$ and $108 \pm 15 \mathrm{MeV} / c^{2}$ for the $X(2370)$ respectively. For the $X(1835)$, the mass is consistent with the BESII result, but the width is significantly larger. 


\section{Observation of $X(1870) \rightarrow a_{0} \pi$ in $J / \psi \rightarrow \omega \pi^{+} \pi^{-} \eta$ decays}

For $J / \psi \rightarrow \omega \pi^{+} \pi^{-} \eta$ decays, as shown in Fig.4, in the $\pi^{+} \pi^{-} \eta$ mass spectrum of Fig 4 (b), besides the $\eta^{\prime}$, there are clear $f_{1}(1285), \eta(1405)$ a structure the $X(1870)$. Fig.4.(c) shows all of the three structures decay primarily via $a_{0}(980) \pi$, and the fitting yields the mass and width are $18373 \pm 11$ and $82 \pm 19 \mathrm{MeV} / c^{2}$ for the $X(1870)$ with significance of $7.7 \sigma$
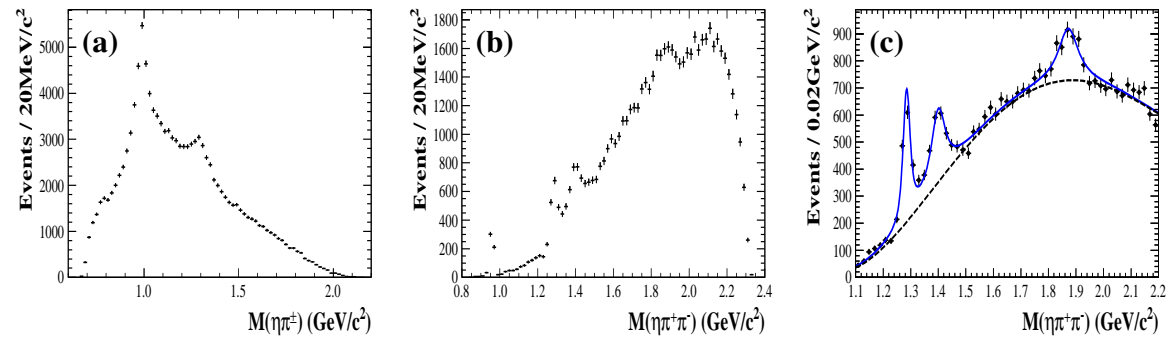

Figure 4: The selected $J / \psi \rightarrow \omega \pi^{+} \pi^{-} \eta$ events: (a) The combined $\eta \pi^{+}$and $\eta \pi^{-}$mass spectrum; (b) The $\eta \pi^{+} \pi^{-}$mass spectrum; (c) The $\eta \pi^{+} \pi^{-}$mass spectrum fitting with $a_{0}$ selection;.

\section{Summary}

In summary, the $p \bar{p}$ mass threshold enhancement $X(1860)$ is confirmed in $J / \psi$ radiative decay, and no obvious similar structure is observed in $\psi^{\prime}$ radiative decay. The $\mathrm{X}(1835)$ is confirmed in $J / \psi \rightarrow \gamma \pi^{+} \pi^{-} \eta^{\prime}$, and two new resonances, $X(2120)$ and $X(2370)$ are observed with significance larger than $7.2 \sigma$ and $6.7 \sigma$ respectively. A new process $J / \psi \rightarrow \omega X(1870) \rightarrow \omega a_{0} \pi$ is observed. Whether or not the $\mathrm{X}(1860), \mathrm{X}(1835)$ and $\mathrm{X}(1870)$ are the same resonance, still needs further study and PWA is a important technique not only to determine the spin-parities of above three resonances, but also to make more precise measurements on masses, widths and Branch ratios by considering possible interferences among them.

\section{References}

[1] BES Collaboration, J.Z. Bai et al., Phys. Rev. Lett. 91, 022001 (2003).

[2] M.Z. Wang et al., Phys. Rev. Lett. 92, 131801 (2004).

[3] BES Collaboration, M. Ablikim et al., Phys. Rev. Lett. 99, 011802 (2007).

[4] CLEO Collaboration, S.B. Athar et al., Phys. Rev. D 73, 032001 (2006).

[5] BES Collaboration, M. Ablikim et al., Eur. Phys. J. C53, 15, (2008).

[6] A. Datta and P.J. O’Donnell, Phys. Lett. B567, 273 (2003); B. Loiseau and S. Wycech, Phys. Rev. C72, 011001 (2005);

[7] BES Collaboration, M. Ablikim et al., Phys. Rev. Lett. 95, 262001 (2005).

[8] N. Kochelev and D. P. Min, Phys. Lett. B 633, 283-288 (2006).

[9] T. Huang and S. L. Zhu, Phys. Rev. D 73, 014023 (2006).

[10] E. Klempt and A. Zaitsev, Phys. Rep. 454, 1-202 (2007). 